\title{
Treatment outcomes and prognostic factor analyses in pulmonary or hepatic metastatic patients treated with stereotactic body radiation therapy - a single institution experience
}

\author{
Wen-Tso Chuang ${ }^{1}$, Stephen Wan Leung ${ }^{1}$, Chang-Yu Wang ${ }^{1}$, Ming-Hsiang Liou ${ }^{1,2}$, Chun-Kau Tse ${ }^{1}$, \\ Ying-Chen Chang ${ }^{1}$
}

${ }^{1}$ Department of Radiation Oncology, Yuan's General Hospital, Kaohsiung, Taiwan; ${ }^{2}$ Department of Medical Imaging and Radiological Sciences, Kaohsiung Medical University, Kaohsiung, Taiwan

Contributions: (I) Conception and design: SW Leung, CY Wang; (II) Administrative support: SW Leung, CY Wang, MH Liou, CK Tse, YC Chang; (III) Provision of study materials or patients: SW Leung, CY Wang; (IV) Collection and assembly of data: WT Chuang, MH Liou, CK Tse, YC Chang; (V) Data analysis and interpretation: WT Chuang, SW Leung, CY Wang, MH Liou; (VI) Manuscript Writing: All authors; (VII) Final Approval of Manuscript: All authors.

Correspondence to: Stephen Wan Leung, MD. Department of Radiation Oncology, Yuan's General Hospital, No. 136, Siwei 4th Rd., Lingya Dist., Kaohsiung City 802, Taiwan. Email: wanleung432@gmail.com.

\begin{abstract}
Background: Stereotactic body radiation therapy (SBRT) is a non-invasive treatment option for metastatic cancer. The purpose of our study was to investigate the efficacy and safety of SBRT for patients with pulmonary or hepatic metastases.

Methods: We retrospectively reviewed patients treated with SBRT between 2013 and 2019. Previous local or systemic treatments were allowed. Our end points were the outcomes in terms of local control (LC) of treated metastases, overall survival (OS), and treatment toxicities. The significance of each prognostic factor was estimated using survival analysis.

Results: Fifty-one patients and 181 metastatic lesions were analyzed. The median follow-up time was 18 months and the median patient age was 60 years old. The predominant origins of primary cancers include hepatocellular carcinoma (HCC) (21.6\%) and colorectal cancer (19.6\%), followed by breast cancer (15.7\%), nonsmall cell lung cancer (NSCLC) (7.8\%), nasopharyngeal cancer (NPC) (7.8\%), and others (21.6\%). The most common prescribed doses were $50 \mathrm{~Gy}$ in 5 fractions (62.4\%) and $60 \mathrm{~Gy}$ in 5 fractions (31.5\%). The 1- and 2-year LC rates were $95.4 \%$ and $85.8 \%$. The 1 - and 2 -year OS rate were $72.9 \%$ and $45.1 \%$ while the median OS was 24 months. The HCC origin group had worse LC (HR 3.12, P=0.039) and more than 3 treated lesions (HR 3.28, $\mathrm{P}=0.008$ ) was negatively impacting the OS. In no events the acute or late toxicities were more than grade 3.
\end{abstract}

Conclusions: The results of our study suggest that SBRT offers a feasible treatment with low toxicities and achieves high rates of LC for pulmonary or hepatic metastases.

Keywords: Stereotactic body radiation therapy (SBRT); metastases; metastasis; pulmonary; hepatic

Received: 23 October 2020; Accepted: 09 February 2021; Published: 30 March 2021.

doi: $10.21037 /$ tro-20-56

View this article at: http://dx.doi.org/10.21037/tro-20-56

\section{Introduction}

Cancer patients with distant metastasis have unfavorable prognoses, and systemic therapies have been the mainstream treatments for decades. Surgical resection and radiofrequency ablation have been considered as local curative options for limited metastatic lesions. With complete surgical resection of all metastatic lesions, the 5 -year overall survival (OS) rate has been reported between $20 \%$ to $50 \%$ in patients with pulmonary metastasectomy and around $40 \%$ in patients with hepatic metastasectomy $(1,2)$. 
However, some patients may not be candidates for surgical resection due to comorbidities or the extent and location of the metastatic lesions. With improvements of radiotherapy technology, stereotactic body radiation therapy (SBRT) has played an important role in local therapy of non-invasive nature. It has been reported that there was no significant difference on OS between SBRT and metastasectomy in pulmonary metastases (3). Compared to conventional radiotherapy, SBRT benefits from steeper dose gradients, higher biological equivalent effectiveness, and relatively less toxicities. SBRT has also been recognized as one of the curative treatment options in early stage and small size non-small cell lung cancer (NSCLC) and hepatocellular carcinoma (HCC) $(4,5)$, especially for medically inoperable patients.

In studies regarding SBRT to metastatic cancer, majorities of published studies were focusing on oligometastases. The concept of oligometastases described the intermediate state between localized to widely spread metastatic (6). It is generally accepted that there are $1-3$ or 1-5 metastatic lesions in oligometastases $(7,8)$. Up to date, there were limited data of randomized controlled trials concerning SBRT on oligometastatic cancers. The SABRCOMET trial, a randomized phase 2 trial, demonstrated that progression free survival (PFS) was doubled with stereotactic ablative radiotherapy (SABR) in addition to standard of care (SOC) compared to SOC alone, and the tendency of improving OS was found (9). A small prospective randomized trial was implemented to evaluate the efficacy of the addition of SABR to maintenance chemotherapy. Improved PFS was found in the arm of combined SABR and maintenance chemotherapy, and the trial was stopped early due to the evidence of statistic improvements (10).

Lung and liver are common metastatic organs in clinical presentation. Previous studies demonstrated that with SBRT, 2-year OS and local control (LC) rate could reach above $50 \%$ and $80 \%$ respectively in patients with oligometastatic pulmonary lesions (11-13). The 5-year OS and LC were approximately $30 \%$ and $80 \%(12,13)$. For hepatic oligometastases, estimated 2-year OS and LC could reach above $55 \%$ and $60 \%$ with SBRT (14-16).

The aim of our study is to evaluate the impact of SBRT on LC, OS, and prognostic factors that affect the patients' LC and OS by different clinical presentations of pulmonary or hepatic metastases.

We present the following article in accordance with the STROBE reporting checklist (available at http://dx.doi. org/10.21037/tro-20-56).

\section{Methods}

\section{Study design and participants}

All patients who consecutively received SBRT for metastatic pulmonary or hepatic lesions from any primary site between October 2013 and July 2019 were reviewed. The study was conducted in accordance with the Declaration of Helsinki (as revised in 2013). The study was approved by institutional medical ethics committee of Yuan's General Hospital (No.: 20181113B). The individual consent for this retrospective analysis was waived. The medical records, including patients' demographics, tumor characteristics, radiation treatments, radiological images, systemic medication, were collected. Patients who had recurrent metastatic lesions which were previously treated with surgery or chemotherapy but not with SBRT were also eligible.

\section{Treatment modalities and SBRT procedures}

As patients underwent computed tomography (CT) simulation scans, they were all immobilized with customized vacuum body molds and thermoplastic masks. For respiratory motion control, we utilized either the deep inspiratory breath hold (DIBH) techniques with the Active Breathing Coordinator (ABC) device (Elekta $A B$, Stockholm, Sweden) or four-dimensional (4D) CT with the free-breathing (FB) method under abdominal compression. The selection of these 2 respiratory motion management techniques was based on the patient's performance status and the compliance of the use of the $\mathrm{ABC}$ device.

For treatment planning, the gross tumor volume (GTV) was defined as the visible tumor on the reference CT imaging from all available fused images [CT, magnetic resonance imaging (MRI), or positron emission tomography] by using MIM software version 5.1 (MIMvista Corp., Cleveland, OH). No additional margins were added for possible microscopic extensions and thus clinical target volume (CTV) is identical to GTV in our treatments. For DIBH technique, a $5 \mathrm{~mm}$ radial and a $10 \mathrm{~mm}$ craniocaudal margins were added from CTV to planning target volume (PTV). For FB technique with 4DCT in pulmonary treatments, the internal target volume (ITV) was delineated with the guidance of maximal intensity projection (MIP) image. Our policy for hepatic metastatic lesions was primarily using DIBH technique. However, there were 4 patients treating hepatic lesions with $\mathrm{FB}$ and abdominal compression due to old age and poor compliance to the ABC device. For their treatments, we contoured the GTV 
on each of 10 phases of 4D CT images and combined these GTVs to generate the ITV by the MIM software. We added $5 \mathrm{~mm}$ margins in all directions from ITV to PTV for patients with FB technique, and the average projection images were utilized for dose calculation. The adjacent organs at risk (OARs) were also contoured. For dose prescriptions and constrains, the volume of the PTV receiving $100 \%$ of the prescription dose was above $95 \%$, and the maximum dose of PTV would be generally less than $110 \%$ of the prescription dose. The prescription dose to PTV was mostly at the range between 50 Gy in 5 fractions and $60 \mathrm{~Gy}$ in 5 fractions, and might be adjusted due to the previous irradiation.

All treatments were planned optimally with Pinnacle treatment planning system version 9.8 (Philips Medical, Madison, WI), and the patients were treated with the linear accelerator, "Infinity ${ }^{\mathrm{TM}}$ (Elekta AB, Stockholm, Sweden), by using volumetric-modulated arc therapy and flattening filter free mode with 6 mega-voltage energy. The treatments were scheduled 2 times per week. Image-guided radiotherapy (IGRT) with kilo-voltage cone beam CT images and the Hexapod Robotic Couch (Elekta AB, Stockholm, Sweden) were utilized for the localization and the correction of the positions.

\section{Follow-up and treatment evaluation}

After the SBRT treatments were completed, the patients were evaluated in outpatient visits 1 month after the last treatment day, every 3 months in the first 2 years, and then every 4 to 6 months thereafter. At each follow-up outpatient contact, both clinical examination and toxicities were assessed. The treatment response was evaluated by serial contrast-enhanced spiral CT or MRI scans 4 to 8 weeks after the end of SBRT and the follow-up scans were performed with 3 to 6 months intervals. We utilized the Response Evaluation Criteria in Solid Tumors 1.1 (RECIST 1.1) and classified the tumor response as complete response, partial response, progressive disease, stable disease (17).

Follow-up period was defined as starting from the end date of the SBRT treatment. The primary endpoint of our study was LC. The secondary endpoints included OS and treatment toxicities. Toxicities which occurred during the treatments and within 3 months after the treatments were considered acute toxicities. Late toxicities were considered as toxicities events happening beyond 3 months after the treatments. Treatment toxicities were recorded according to common terminology criteria for adverse events (CTCAE) scale, version 4.0.3.

\section{Statistics}

The following variables were analyzed with descriptive statistics: age, gender, performance status, primary tumor origins, previous regional irradiation (the same organ irradiated previously beyond the SBRT treatment targets), metastases status (synchronous or metachronous), SBRT irradiation dose, number of treatment fractions, size of the treated lesions, motion management of treatments, and treatment-related toxicities. Metastases were considered synchronous if metastatic disease was present within six months of the primary cancer diagnosis, and metachronous if present beyond six months after the primary cancer diagnosis. The time to event endpoints were calculated from the last day of SBRT. Local recurrence was defined as either reappearance after complete remission, or progression in beyond 3 months after initial partial response to SBRT within the irradiated volumes in follow-up CT or MRI scans. The event of OS was defined as death from any cause. Survival rates were estimated by the Kaplan-Meier method and groups were compared with the log-rank test. $\mathrm{P}$ values smaller than 0.05 were considered statistically significant. Univariate and multivariate Cox regression analyses were utilized to assess the relationships between the outcomes and possible prognostic variables. All statistical analyses were done by using SPSS version 21 software (IBM Corp., Armonk, N.Y., USA).

\section{Results}

\section{Patient characteristics}

From October 2013 to July 2019, there were 60 patients who received SBRT for pulmonary or hepatic metastatic lesions. 9 patients were excluded for having 2 types of primary malignancy or having no follow-up images performed after SBRT. Fifty-one patients and 181 metastatic lesions were analyzed, and the baseline characteristics are listed in Table 1. The median age was 60 years old (range, 27-87 years). The ECOG performance statuses of majority patients were scored 1 . The median follow-up time was 27 months for the alive 22 patients (range, 1-65 months) while the median follow-up time was 18 months for overall 51 patients (range, 1-65 months). The origins of primary cancers included HCC (21.6\%) and colorectal cancer (CRC) (19.6\%), followed by breast cancer (15.7), NSCLC (7.8\%), nasopharyngeal cancer (NPC) $(7.8 \%)$, and others (21.6\%). Thirty-four patients $(66.7 \%)$ received SBRT for having only 1 to 3 lesions. Metachronous disease was 
Table 1 Patient characteristics

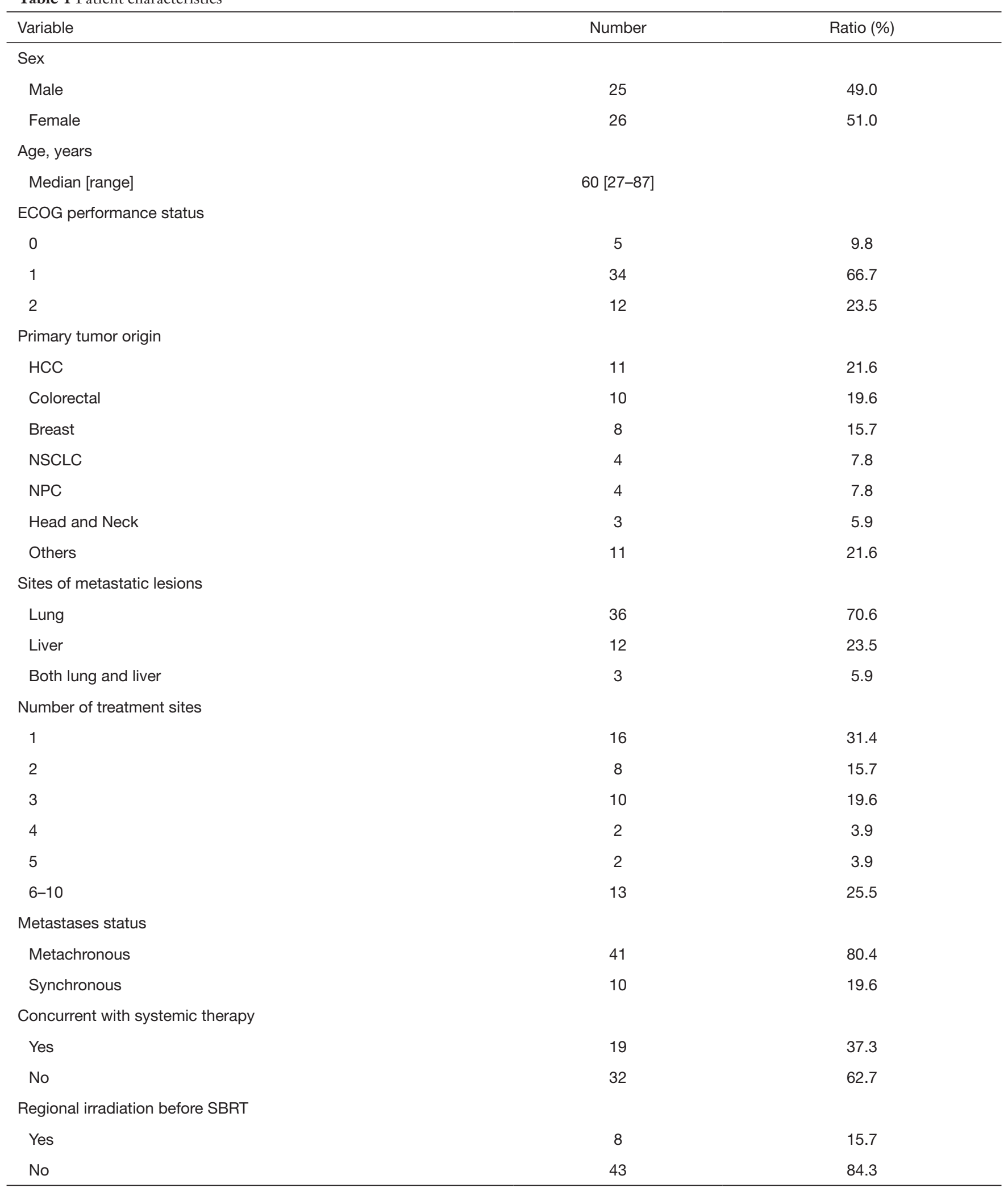

ECOG, Eastern Cooperative Oncology Group; HCC, hepatocellular carcinoma; NSCLC, non-small cell lung cancer; NPC, nasopharyngeal carcinoma; SBRT, stereotactic body radiation therapy. 
Table 2 Stereotactic body radiation therapy (SBRT) treatment characteristics

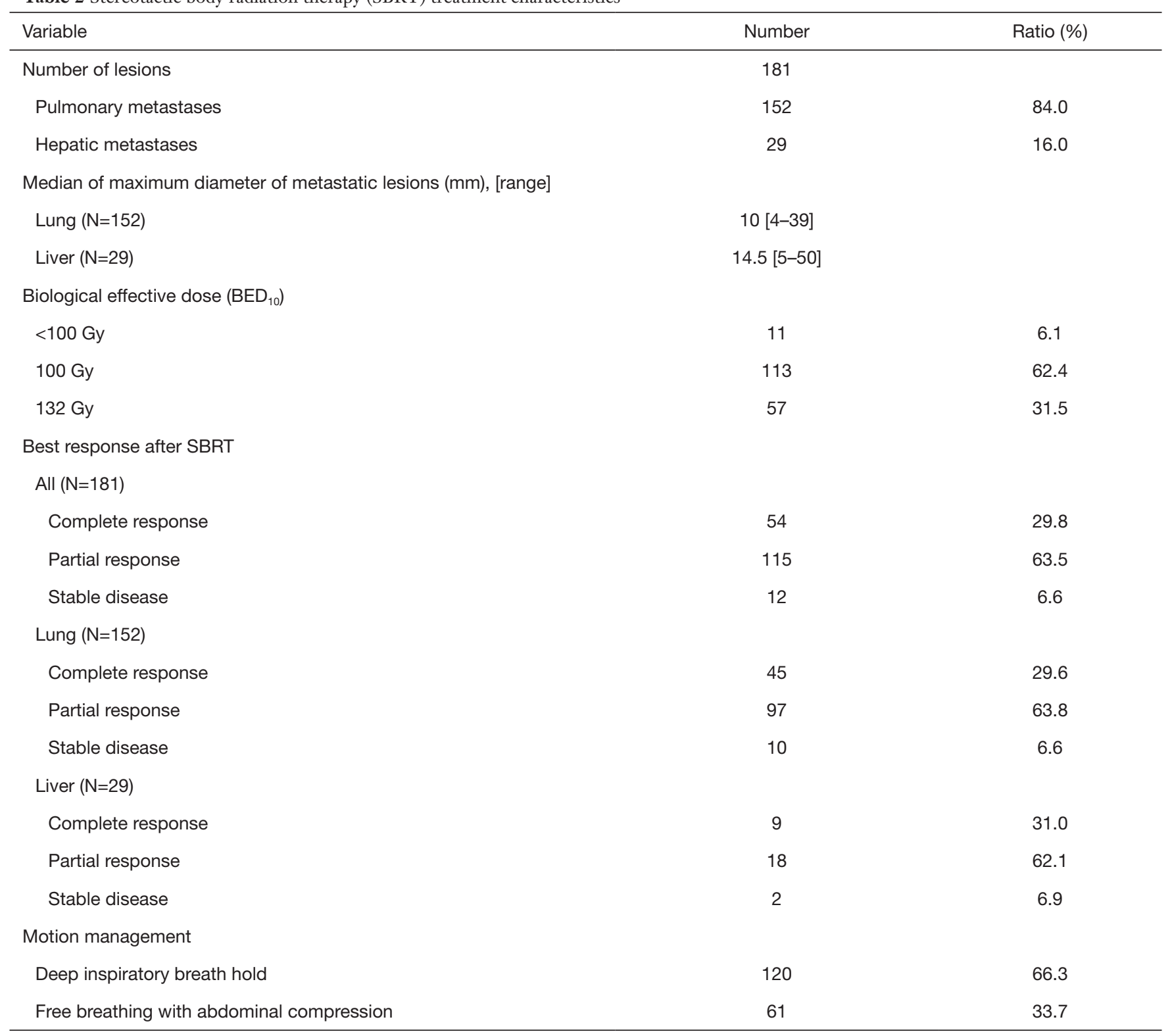

the predominant metastatic status in our patients. Except the SBRT treatment lesions, the primary cancers were all controlled before the patients received SBRT.

\section{Treatment characteristics}

As listed in Table 2, totally 152 pulmonary and 29 hepatic metastatic lesions were treated. The median diameter of the lesions was $10 \mathrm{~mm}$ (range, 4-39 $\mathrm{mm}$ ) for the pulmonary lesions and $14.5 \mathrm{~mm}$ (range, $5-50 \mathrm{~mm}$ ) for the hepatic lesions. The most common prescribed doses were $50 \mathrm{~Gy}$ in 5 fractions $(62.4 \%)$ and 60 Gy in 5 fractions $(31.5 \%)$. The biological effective dose (BED, in $\alpha / \beta=10)$ of the former is 100 Gy while the $\mathrm{BED}_{10}$ of the latter is $132 \mathrm{~Gy}$. It was observed that 8 pulmonary lesions and 3 hepatic lesions within 8 patients were treated with $\mathrm{BED}_{10}$ less than $100 \mathrm{~Gy}$. The reasons for adjustments of the prescribed doses were due to previous regional irradiation in 8 lesions, 2 lesions with complete remission during the third fraction of the treatment, and 1 pulmonary lesion located near the hilar. 


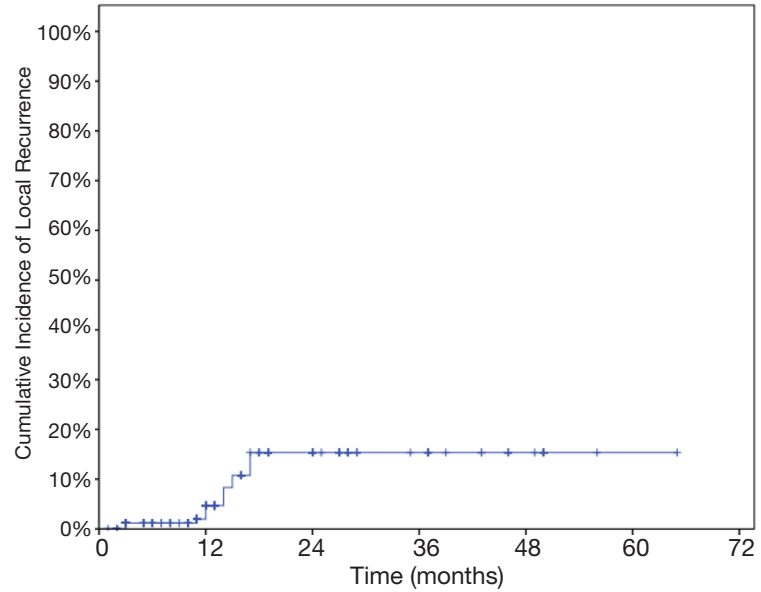

Figure 1 Cumulative incidence of local recurrence after SBRT for pulmonary/hepatic metastases $(\mathrm{N}=181)$. SBRT, stereotactic body radiation therapy.

\section{Treatment efficacy}

Regarding the responses at 3 months after the SBRT treatments were completed, the rate of complete response, partial response, and stable disease in all lesions were $30.0 \%, 63.3 \%$, and $6.7 \%$ respectively. The treatment responses were almost similar for pulmonary and hepatic lesions.

Local recurrence was observed in 14/181 (7.5\%) metastatic lesions, including 10 pulmonary lesions and 4 hepatic lesions in 6 patients. There were 2 HCC patients having 5 local recurrent lesions, followed by 2 CRC patients having 3 local recurrent lesions, 1 NPC patient having 3 local recurrent lesions, and 1 breast cancer patient having 3 local recurrent lesions. Among these 14 lesions, the median time from the SBRT treatments to local recurrence was 14 months. The controlled recurrent pulmonary lesions were noted in 1 patient who received salvage metastasectomy. 2 patients had progressed disease despite of local treatment, and the other 3 patients had no further treatments.

The LC rates were $95.4 \%, 85.8 \%$ at 12 months and 24 months respectively (Figure 1). At the analysis of correlation between risk factors and LC, only HCC origin was found to have impacts on the control of treated metastases [HR 3.12; 95\% confidence interval (CI), 1.06-9.22, $\mathrm{P}=0.039$ ] (Table 3, Figure 2). In the analysis of 152 lung treated lesions, HCC origin had the tendency with worse LC. No significant impacts were revealed whether the treated lesions were in central or peripheral locations in pulmonary metastases.

At last follow-up, 29 patients (56.9\%) had died. The 1- and 2-year OS rate were $72.9 \%$ and $45.1 \%$ while the median OS was 24 months (95\% CI, 13.97-34.03) (Figure 3). At univariate analysis, HCC origin (HR 2.82; 95\% CI, 1.21-6.60, $\mathrm{P}=0.011$ ) and $>3$ treated lesions (HR 4.57; 95\% CI, 2.16-9.68, $\mathrm{P}<0.001)$ were negatively impacting on OS. Patients treated with $\mathrm{BED}_{10} \geq 132$ Gy (HR 0.34, 95\% CI, $0.16-0.83, \mathrm{P}=0.017$ ) had better OS rate (Table 4). Only $>3$ treated lesions (HR 3.28; 95\% CI, 1.37-7.87, P=0.008) was negatively impacting on OS in multivariate analysis (Figure 4).

\section{Treatment toxicity}

The acute toxicities after the SBRT treatments were generally mild except 1 patient had grade 3 leukocytopenia with concurrent chemotherapy during the treatment for hepatic lesions (Table 5). In patients treating for pulmonary lesions, the overall rate of any grade 1 or 2 late toxicities was $82.1 \%$. The most common toxicities included radiation pneumonitis and segmental atelectasis (Table 6). There were 2 patients had grade $\geq 3$ late toxicities. One patient had grade 3 spontaneous pneumothorax and needed hospitalization for treatment. The other patient had grade 3 radiation pneumonitis and required oxygen supplements for several months. In patients treating for hepatic lesions, there were no grade $\geq 2$ late toxicities observed and only 2 patients having grade 1 late toxicities. One patient had grade 1 radiation pneumonitis and the other patient had grade 1 pulmonary atelectasis.

\section{Discussion}

The study reports our experience of SBRT for the treatments of metastatic pulmonary or hepatic tumors. After a median follow-up time of 18 months for all patients, we demonstrated high rates of LC after SBRT without causing major toxicities. Even across in different histology types, our treatments achieved the good LC rates of $95.4 \%$ at 1 year and $85.8 \%$ at 2 years for pulmonary or hepatic metastatic lesions. He et al, reported 40 CRC patients with 57 lung or liver metastatic sites treated with radiotherapy, and the LC rates were $63.2 \%, 24.6 \%, 16.9 \%$ at 1,3 and 5 years (18). Fumagalli et al. also investigated 90 patients from various origins with 26 pulmonary and 113 hepatic metastatic lesions. Their LC rates at 1 and 2 years were $84.5 \%$ and $66.1 \%$ respectively (19). Other large studies investigated pulmonary oligometastases and showed the LC rates at 2 years could be achieved above $80 \%(3,11,12,13,20)$. For hepatic oligometastases, various outcomes of LC 
Table 3 Univariate and multivariable analysis for local control

\begin{tabular}{|c|c|c|c|c|}
\hline Factors & \multicolumn{2}{|l|}{ Univariate } & \multicolumn{2}{|c|}{ Multivariate } \\
\hline Age $>65$ & $0.027(0.000-3.082)$ & 0.135 & - & \\
\hline Sex, male & $0.709(0.247-2.031)$ & 0.522 & - & \\
\hline Performance status & $0.890(0.387-2.048)$ & 0.784 & - & \\
\hline Tumor origin, $\mathrm{HCC}$ & $3.124(1.058-9.222)$ & $0.039^{*}$ & - & - \\
\hline Metastatic sites, liver/lung & $1.543(0.768-3.098)$ & 0.223 & - & \\
\hline Number of treated lesions, $>3$ & $0.708(0.243-2.063)$ & 0.526 & - & \\
\hline Previous local therapy & $1.743(0.404-1.743)$ & 0.404 & - & \\
\hline Size of treated lesion, $\geq 3 \mathrm{~cm}$ & $0.469(0.042-225.763)$ & 0.042 & - & - \\
\hline Post-SBRT chemotherapy & $0.470(0.162-1.363)$ & 0.165 & - & \\
\hline Metastatic status, metachronous & $0.511(0.135-1.940)$ & 0.324 & - & \\
\hline $\mathrm{BED}_{10}$ Gy, $\geq 132$ Gy & $0.372(0.103-1.338)$ & 0.130 & - & \\
\hline Respiratory management, DIBH vs. FB & $1.046(0.290-3.777)$ & 0.945 & - & \\
\hline
\end{tabular}

*, $\mathrm{P}<0.05$. HR, hazard ratio; $\mathrm{Cl}$, confidence interval; HCC, hepatocellular carcinoma; SBRT, stereotactic body radiation therapy; BED, biological effective dose; $\mathrm{DIBH}$, deep inspiration breath hold; $\mathrm{FB}$, free breathing.

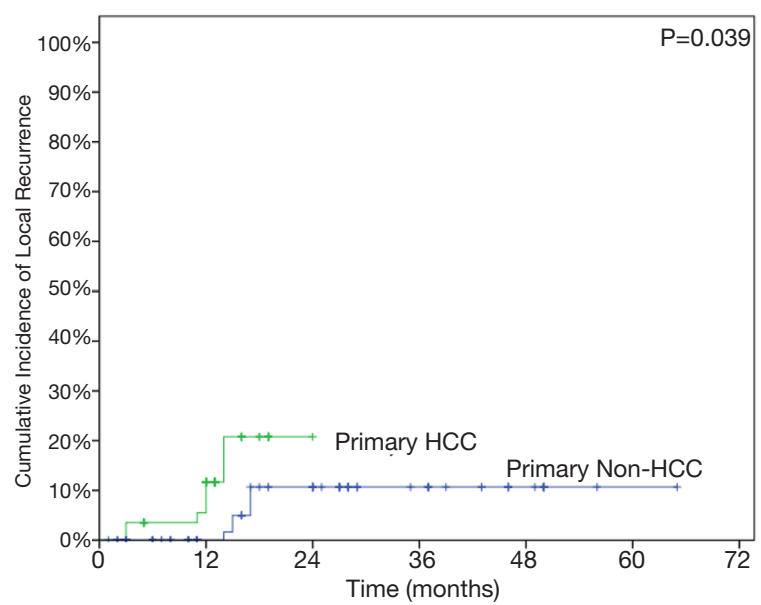

Figure 2 Cumulative incidence of local recurrence stratified by the primary tumor origin.

were reported, achieving around $60 \%$ at 2 years (14-16). Our results are compatible to the above studies, and the favourable LC rates suggest that our treatment represent robust practice.

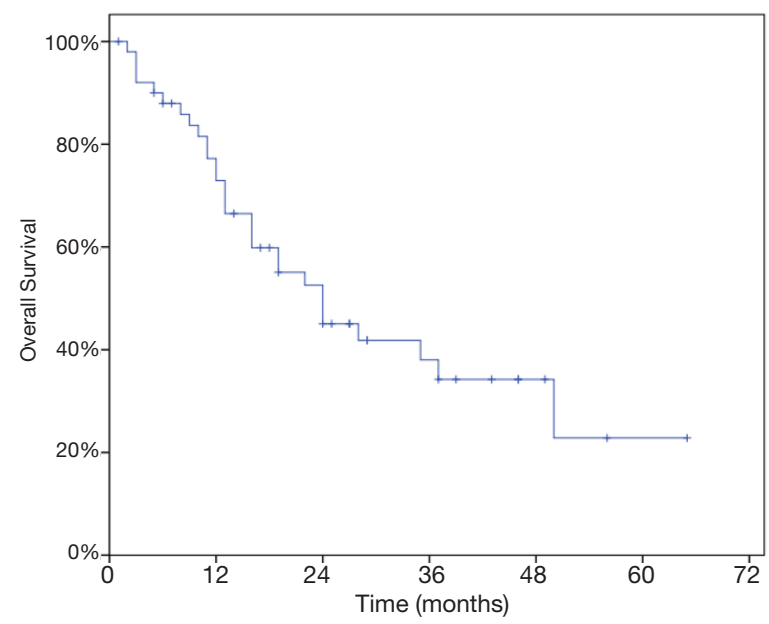

Figure 3 Overall survival following SBRT for pulmonary/hepatic metastases $(\mathrm{N}=51)$. SBRT, stereotactic body radiation therapy.

Regarding the whole group, the OS rates were $72.9 \%$ and $45.1 \%$ at 1 and 2 years in our study. These results are comparable to the studies we reviewed. Petrelli et al. conducted a systematic review regarding hepatic metastases 
Table 4 Univariate and multivariable analysis for overall survival.

\begin{tabular}{|c|c|c|c|c|}
\hline Factors & \multicolumn{2}{|c|}{ Univariate } & \multicolumn{2}{|c|}{ Multivariate } \\
\hline Age $>65$ & $1.413(0.624-3.199)$ & 0.396 & - & \\
\hline Sex, male & $1.056(0.732-1.524)$ & 0.766 & - & \\
\hline Performance status & $0.906(0.465-1.765)$ & 0.953 & - & \\
\hline Primary tumor origin, $\mathrm{HCC}$ & $2.824(1.209-6.598)$ & $0.011^{*}$ & $1.510(0.588-3.882)$ & 0.392 \\
\hline Metastatic sites, hepatic/pulmonary & $0.887(0.463-1.702)$ & 0.794 & - & \\
\hline Number of treated lesions, $>3$ & 4.567 (2.155-9.678) & $<0.001^{\star \star}$ & $3.280(1.367-7.872)$ & $0.008^{*}$ \\
\hline Previous local therapy & $0.861(0.580-1.279)$ & 0.450 & - & \\
\hline Size of treated lesion, $\geq 3 \mathrm{~cm}$ & $2.349(0.684-8.074)$ & 0.156 & - & \\
\hline Treatment after local progression & $2.046(0.615-6.802)$ & 0.225 & - & \\
\hline Post-SBRT chemotherapy & $1.466(0.689-3.118)$ & 0.310 & - & \\
\hline Metastatic status, metachronous & $0.497(0.210-1.181)$ & 0.100 & - & \\
\hline Local recurrence after SBRT & $1.161(0.401-3.357)$ & 0.780 & - & \\
\hline $\mathrm{BED}_{10} \mathrm{~Gy}, \geq 132$ Gy & $0.336(0.160-0.834)$ & $0.017^{*}$ & $0.576(0.234-1.407)$ & 0.226 \\
\hline
\end{tabular}

*, $\mathrm{P}<0.05$; ** $\mathrm{P}<0.001$. HR, hazard ratio; Cl, confidence interval; HCC, hepatocellular carcinoma; BED, biological effective dose; SBRT, stereotactic body radiation therapy.

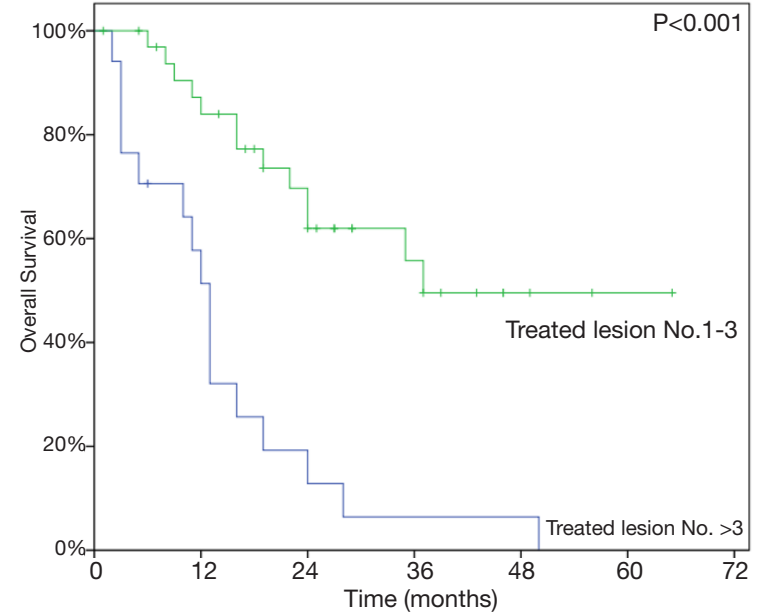

Figure 4 Overall survival stratified by the number of treated lesions. from CRC patients. The pooled 1- and 2-year OS were $67.18 \%$ (95\% CI, 42.1-92.2) and 56.5\% (95\% CI, $36.7-$ 76.2), respectively (14). A systematic review was reported by Alongi et al. regarding pulmonary metastatic lesions treated with SBRT. The 1-year and 2-year OS rates varied from $76 \%$ to $98 \%$ and $31 \%$ to $76 \%$, respectively (21). For comparison between pulmonary metastasectomy and SBRT, Lee et al. reported that 1- and 2-year OS rates were $95 \%$ and $81.8 \%$ in the metastasectomy group and $79.5 \%$ and $68.2 \%$, in the SBRT group. However, the treatment modalities did not affect OS in multivariate analysis (3). Widder et al. also found there were no significant differences between pulmonary metastasectomy and SBRT. The estimated 1-, 2-, and 5-year OS rates were 87\%, 74\%, $41 \%$ in the metastasectomy group and $98 \%, 86 \%, 49 \%$ in the SBRT group (22). Our outcomes are quite inferior to 
Table 5 Acute toxicities in SBRT treatment

\begin{tabular}{|c|c|c|c|c|}
\hline Toxicity grade & \multicolumn{4}{|c|}{ No. of events } \\
\hline \multicolumn{5}{|l|}{ Pulmonary } \\
\hline Cough & 13 & 4 & 0 & 0 \\
\hline Dyspnea & 7 & 3 & 0 & 0 \\
\hline Chest pain & 2 & 0 & 0 & 0 \\
\hline \multicolumn{5}{|l|}{ Hepatic } \\
\hline Fatigue & 4 & 0 & 0 & 0 \\
\hline Vomiting & 0 & 1 & 0 & 0 \\
\hline Leukocytopenia & 0 & 0 & 1 & 0 \\
\hline
\end{tabular}

SBRT, stereotactic body radiation therapy.

Table 6 Late toxicities in SBRT treatment

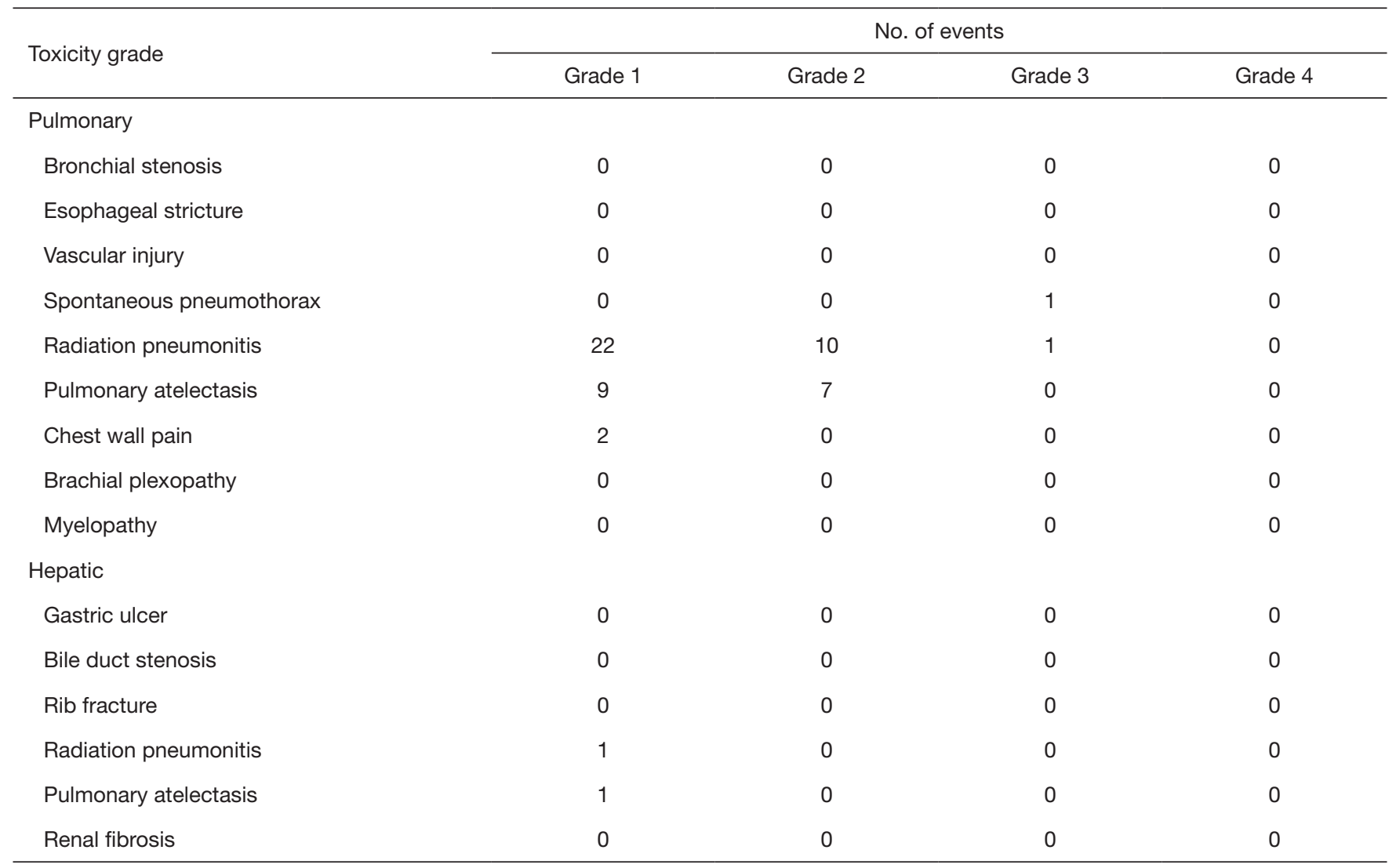

SBRT, stereotactic body radiation therapy. 
patients receiving metastasectomy in reviewed literatures: it might be because our patients were not suitable to operation due to comorbidities. Considering the general negative selection of patients which were not considered as surgical candidates, our survival outcomes appear to be reasonable.

Metastatic tumors with primary HCC origin were associated with worse outcomes in our study. In HCC patients, the 1 -year OS rate was $70.7 \%$ and the 1 -year LC rate was $89 \%$. Classically, HCC was considered as a radioresistant tumor, and there were very few effective systemic treatments for primary hepatic malignancy. The poor outcomes in OS might be a consequence of the patient characteristics in our study: HCC is the most common primary origin and there were 11 patients $(21.6 \%)$ in our study. This relationship was not widely reported, though more failures from the liver origin were reported by $\mathrm{Oh}$ et al. (23). To our knowledge, relatively few HCC patients were reported in previous studies of SBRT treatments for metastases, and the majorities of the studies focused on CRC patients. Worse LC rates were found to be associated with CRC origin in pulmonary or hepatic oligometastases (15,24-27). However, CRC origin was reported to have favorable OS in both pulmonary $(13,27)$ and hepatic oligometastases (15) in contrast.

Our study represents that the numbers of treated lesions are related to survival outcomes and the worse OS were found in patients who had $>3$ treated lesions. Kinj et al. reported 53 CRC patients treated with 87 pulmonary oligometastases; $\geq 3$ treated metastases had negative impact on both OS and LC comparing with $<3$ treated lesions (28). A few reports also revealed multiple treated lesions had worse survivals than single treated lesion $(18,29,30)$. The relationship might be a consequence of higher tumor burdens, thus poorer prognosis.

Another intriguing factor is irradiation dose. In our study, $93.9 \%$ of the treated lesions were irradiated with $\mathrm{BED}_{10}$ $\geq 100 \mathrm{~Gy}$, and the treatments had good LC rate of $85.8 \%$ at 2 years. Some studies demonstrated that the irradiation dose did affect the treatment outcomes. Aman Sharma et al. demonstrated that $\mathrm{BED}_{10} \geq 100 \mathrm{~Gy}$ was predictive to better OS and LC in CRC patients treated for pulmonary oligometastases (29). Another systemic review conducted by Petrelli et al. showed the linear correlation between SBRT BED dose to $\mathrm{LC}(\mathrm{P}=0.001, \mathrm{R}=0.47)$ and $\mathrm{OS}(\mathrm{P}=0.001$, $\mathrm{R}=0.29)$ in CRC patients with hepatic metastases (14). Andratschke $e t$ al. also reported the treatment outcomes of 474 patients with 623 liver metastases. Better LC rate of above $80 \%$ can be achieved if $\mathrm{BED}_{10}$ is prescribed greater than 150 Gy (15).

Generally, our study is limited by the retrospective design, the heterogeneity of primary cancer origins, the small population size, and relatively short follow-up time that recurrence might be underestimated. The weakness of selection bias is implicit as other retrospective SBRT studies. A comprehensive statistical analysis was impossible due to the relative small sample size and few negative events.

In conclusion, the results of our study suggest that SBRT offers a feasible treatment with low toxicities and achieves high rates of LC for pulmonary or hepatic metastases. HCC origin correlates to LC while more than 3 treated lesions are predictive to OS in our study. However, more prospective studies are needed for the evaluation of optimal fractionation dose and for the considerations of patient candidates in the future.

\section{Acknowledgments}

Funding: This investigation was supported by grants from the Yuan's General Hospital (YGH-19-016).

\section{Footnote}

Reporting Checklist: The authors have completed the STROBE reporting checklist. Available at http://dx.doi. org/10.21037/tro-20-56

Conflicts of Interest: All authors have completed the ICMJE uniform disclosure form (available at http://dx.doi. org/10.21037/tro-20-56). The authors have no conflicts of interest to declare.

Ethical Statement: The authors are accountable for all aspects of the work in ensuring that questions related to the accuracy or integrity of any part of the work are appropriately investigated and resolved. The study was conducted in accordance with the Declaration of Helsinki (as revised in 2013). This retrospective study was approved by institutional medical ethics committee of Yuan's General Hospital (No.: 20181113B). The individual consent for this retrospective analysis was waived.

Open Access Statement: This is an Open Access article distributed in accordance with the Creative Commons Attribution-NonCommercial-NoDerivs 4.0 International License (CC BY-NC-ND 4.0), which permits the non- 
commercial replication and distribution of the article with the strict proviso that no changes or edits are made and the original work is properly cited (including links to both the formal publication through the relevant DOI and the license). See: https://creativecommons.org/licenses/by-nc-nd/4.0/.

\section{References}

1. Petrella F, Diotti C, Rimessi A, et al. Pulmonary metastasectomy: an overview. J Thorac Dis 2017;9:S1291-S1298.

2. Kanas GP, Taylor A, Primrose JN, et al. Survival after liver resection in metastatic colorectal cancer: review and meta-analysis of prognostic factors. Clin Epidemiol 2012;4:283-301.

3. Lee YH, Kang KM, Choi HS, et al. Comparison of stereotactic body radiotherapy versus metastasectomy outcomes in patients with pulmonary metastases. Thorac Cancer 2018;9:1671-9.

4. Chang JY, Senan S, Paul MA, et al. Stereotactic ablative radiotherapy versus lobectomy for operable stage I nonsmall-cell lung cancer: a pooled analysis of two randomised trials. Lancet Oncol 2015;16:630-7.

5. Bujold A, Massey CA, Kim JJ, et al. Sequential phase I and II trials of stereotactic body radiotherapy for locally advanced hepatocellular carcinoma. J Clin Oncol 2013;31:1631-9.

6. Hellman S, Weichselbaum RR. Oligometastases. J Clin Oncol 1995;13:8-10.

7. Milano MT, Katz AW, Zhang H, et al. Oligometastases treated with stereotactic body radiotherapy: long-term follow-up of prospective study. Int J Radiat Oncol Biol Phys 2012;83:878-86

8. Palma DA, Salama JK, Lo SS, et al. The oligometastatic state - separating truth from wishful thinking. Nat Rev Clin Oncol 2014;11:549-57.

9. Palma DA, Olson R, Harrow S, et al. Stereotactic ablative radiotherapy versus standard of care palliative treatment in patients with oligometastatic cancers (SABRCOMET): a randomised, phase 2, open-label trial. Lancet 2019;393:2051-8.

10. Iyengar P, Wardak Z, Gerber DE, et al. Consolidative Radiotherapy for Limited Metastatic Non-Small-Cell Lung Cancer: A Phase 2 Randomized Clinical Trial. JAMA Oncol 2018;4:e173501.

11. Rieber J, Streblow J, Uhlmann L, et al. Stereotactic body radiotherapy (SBRT) for medically inoperable lung metastases-A pooled analysis of the German working group "stereotactic radiotherapy". Lung Cancer 2016;97:51-8.

12. Sharma A, Duijm M, Oomen-de Hoop E, et al. Factors affecting local control of pulmonary oligometastases treated with stereotactic body radiotherapy. Acta Oncol 2018;57:1031-7.

13. Sharma A, Duijm M, Oomen-de Hoop E, et al. Survival and prognostic factors of pulmonary oligometastases treated with stereotactic body radiotherapy. Acta Oncol 2019;58:74-80.

14. Petrelli F, Comito T, Barni S, et al. Stereotactic body radiotherapy for colorectal cancer liver metastases: A systematic review. Radiother Oncol 2018;129:427-34.

15. Andratschke N, Alheid H, Allgäuer M, et al. The SBRT database initiative of the German Society for Radiation Oncology (DEGRO): patterns of care and outcome analysis of stereotactic body radiotherapy (SBRT) for liver oligometastases in 474 patients with 623 metastases. BMC Cancer 2018;18:283

16. Goodman BD, Mannina EM, Althouse SK, et al. Longterm safety and efficacy of stereotactic body radiation therapy for hepatic oligometastases. Pract Radiat Oncol 2016;6:86-95.

17. Eisenhauer EA, Therasse P, Bogaerts J, et al. New response evaluation criteria in solid tumours: revised RECIST guideline (version 1.1). Eur J Cancer 2009;45:228-47.

18. He X, Zhang P, Li Z, et al. Curative-intent radiotherapy in patients with oligometastatic lesions from colorectal cancer: A single-center study. Medicine (Baltimore) 2018;97:e12601.

19. Fumagalli I, Bibault JE, Dewas S, et al. A single-institution study of stereotactic body radiotherapy for patients with unresectable visceral pulmonary or hepatic oligometastases. Radiat Oncol 2012;7:164.

20. Dohopolski MJ, Horne Z, Clump D, et al. Stereotactic Body Radiation Therapy for Pulmonary Oligometastases Arising from Non-lung Primaries in Patients Without Extrapulmonary Disease. Cureus 2018;10:e2167.

21. Alongi F, Mazzola R, Figlia V, et al. Stereotactic body radiotherapy for lung oligometastases: Literature review according to PICO criteria. Tumori 2018;104:148-56.

22. Widder J, Klinkenberg TJ, Ubbels JF, et al. Pulmonary oligometastases: metastasectomy or stereotactic ablative radiotherapy? Radiother Oncol 2013;107:409-13.

23. Oh D, Ahn YC, Seo JM, et al. Potentially curative stereotactic body radiation therapy (SBRT) for single or oligometastasis to the lung. Acta Oncol 2012;51:596-602.

24. Binkley MS, Trakul N, Jacobs LR, et al. Colorectal Histology Is Associated With an Increased Risk of Local 
Failure in Lung Metastases Treated With Stereotactic Ablative Radiation Therapy. Int J Radiat Oncol Biol Phys 2015;92:1044-52.

25. Helou J, Thibault I, Poon I, et al. Stereotactic Ablative Radiation Therapy for Pulmonary Metastases: Histology, Dose, and Indication Matter. Int J Radiat Oncol Biol Phys 2017;98:419-27.

26. Hong JC, Ayala-Peacock DN, Lee J, et al. Classification for long-term survival in oligometastatic patients treated with ablative radiotherapy: A multi-institutional pooled analysis. PLoS One 2018;13:e0195149.

27. Cao C, Wang D, Tian DH, et al. A systematic review and meta-analysis of stereotactic body radiation therapy for colorectal pulmonary metastases. J Thorac Dis

\section{doi: $10.21037 /$ tro-20-56}

Cite this article as: Chuang WT, Leung SW, Wang CY, Liou MH, Tse CK, Chang YC. Treatment outcomes and prognostic factor analyses in pulmonary or hepatic metastatic patients treated with stereotactic body radiation therapy-a single institution experience. Ther Radiol Oncol 2021;5:2.
2019;11:5187-98.

28. Kinj R, Bondiau PY, François E, et al. Radiosensitivity of Colon and Rectal Lung Oligometastasis Treated With Stereotactic Ablative Radiotherapy. Clin Colorectal Cancer 2017;16:e211-e220.

29. Sharma A, Baker S, Duijm M, et al. Prognostic factors for local control and survival for inoperable pulmonary colorectal oligometastases treated with stereotactic body radiotherapy. Radiother Oncol 2020;144:23-9.

30. Yamashita H, Niibe Y, Yamamoto T, et al. Lung stereotactic radiotherapy for oligometastases: comparison of oligo-recurrence and sync-oligometastases. Jpn J Clin Oncol 2016;46:687-91. 